INPLASY

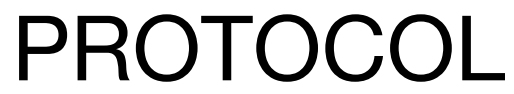

To cite: Sarmento et al.

Depressive Symptoms and

Burnout in Football Players-A

Systematic Review. Inplasy

protocol 202080074. doi:

10.37766/inplasy2020.8.0074

Received: 18 August 2020

Published: 18 August 2020

Corresponding author:

Hugo Sarmento

hg.sarmento@gmail.com

Author Affiliation:

University of Coimbra,

Research Unit for Sport and

Physical Activity, Faculty of

Sport Sciences and Physical

Education

Support: None.

Review Stage at time of this submission: Data analysis.

Conflicts of interest:

The authors declare no

conflicts of interest.

\section{Depressive Symptoms and Burnout in Football Players-A Systematic Review}

Sarmento, $\mathrm{H}^{1}$; Frontini, $\mathrm{R}^{2}$; Marques, $\mathrm{A}^{3}$; Peralta, $\mathrm{M}^{4}$; Saavedra, $\mathrm{NO}^{5}$; Clemente, $\mathrm{FM}^{6}$.

Review question / Objective: This review aimed to systematically review and organize the available literature dedicated to the topic of depression and burnout among football players.

Condition being studied: Depression and burnout in soccer players.

Information sources: A systematic review strategy was conducted according to PRISMA (Preferred Reporting Items for Systematic Reviews and Meta-analyses) guidelines. Electronic databases (Web of Science, Scopus, SPORTdiscus, PubMed, and Psychinfo) were searched (1st July 2020) for relevant publications before up to June 2020. Keywords and synonyms were entered in various combinations (i.e., "Soccer" OR Depression OR "depressive disorder", "depressive disorder" OR burnout OR "mental health" OR "emotional exhaustion").

INPLASY registration number: This protocol was registered with the International Platform of Registered Systematic Review and Meta-Analysis Protocols (INPLASY) on 18 August 2020 and was last updated on 18 August 2020 (registration number INPLASY202080074).

\section{INTRODUCTION}

Review question / Objective: This review aimed to systematically review and organize the available literature dedicated to the topic of depression and burnout among football players.
Rationale: Depression and burnout are often ignored in soccer. Although there have been important systematic reviews regarding the physical health of football players, little research has been devoted to their mental health. Therefore, this review aimed to systematically review and organize the available literature dedicated 
to the topic of depression and burnout among football players.

Condition being studied: Depression and burnout in soccer players.

\section{METHODS}

Search strategy: A systematic review strategy was conducted according to PRISMA (Preferred Reporting Items for Systematic Reviews and Meta-analyses) guidelines. Electronic databases (Web of Science, Scopus, SPORTdiscus, PubMed, and Psychinfo) were searched (1st July 2020) for relevant publications before up to June 2020.

Participant or population: Soccer players.

Intervention: Depression and burnout groups.

\section{Comparator: Control.}

\section{Study designs to be included: Control.}

Eligibility criteria: The publications included met the following criteria: (1) contained relevant data concerning depression and/ or burnout in football players; (2) included male/female football players; and (3) were written in English.

Information sources: A systematic review strategy was conducted according to PRISMA (Preferred Reporting Items for Systematic Reviews and Meta-analyses) guidelines. Electronic databases (Web of Science, Scopus, SPORTdiscus, PubMed, and Psychinfo) were searched (1st July 2020) for relevant publications before up to June 2020. Keywords and synonyms were entered in various combinations (i.e., "Soccer" OR Depression OR "depressive disorder*", "depressive disorder" OR burnout OR "mental health" OR "emotional exhaustion").

Main outcome(s): Depressive symptoms and burnout symptoms.
Quality assessment / Risk of bias analysis: The appraisal tool to assess the quality of cross-sectional studies (AXIS) was used to classify the methodological quality of the articles.

Strategy of data synthesis: The data extracted from the eligible studies were grouped into three categories: (i) General study descriptors (e.g., authors, year of publication and study design). (ii) Description of the study population (e.g., sample size, age, sex, country and competitive level). (iii) data concerning the qualitative synthesis (e.g., outcomes, instruments used to evaluate the symptoms, and main results).

Subgroup analysis: No analysis.

Sensibility analysis: No analysis.

Language: English.

Country(ies) involved: Portugal; Colombia.

Keywords: soccer; sports; depression; mental health; burnout.

Contributions of each author:

Author 1 - Hugo Sarmento - Head of the project; data search; methodological assessment; qualitative synthesis; Writing and revision of the article.

Author 2 - Roberta Frontini - Writing and revision of the article.

Author 3 - Adilson Marques - Writing and revision of the article.

Author 4 - Miguel Peralta - Writing and revision of the article.

Author 5 - Nestor Ordoñez Saavedra Writing and revision of the article.

Author 6 - Filipe Manuel Clemente - Data search; methodological assessment; qualitative synthesis; Writing and revision of the article. 